\title{
APERTURE COUPLED MICROSTRIP SHORT BACKFIRE ANTENNA
}

\author{
Georgi S. Kirov ${ }^{*}$ - Georgi T. Chervenkov* - Chavdar D. Kalchev ${ }^{* *}$
}

\begin{abstract}
A broadband aperture coupled microstrip short backfire antenna is described herein. It consists of a feed part (a microstrip feed line and a coupling slot in a metal ground) and a radiating part with two radiators: a patch antenna and a backfire antenna. The bandwidth widening of the antenna is achieved by use of two resonances: a patch resonance and a backfire resonance. The antenna is designed to operate within the Ku-band. It has a frequency bandwidth of about $15 \%$ and a maximum gain of $11.5 \mathrm{dBi}$. Within the antenna bandwidth the gain and the radiation efficiency have values more than $9 \mathrm{dBi}$ and $82.1 \%$, respectively. The designed antenna has a simple and compact construction and high mechanical and electrical characteristics. It can be used as a single antenna or as an element of microstrip antenna arrays with various applications in the contemporary communication systems.
\end{abstract}

K e y w ords: aperture coupled microstrip antenna, aperture coupled microstrip short backfire antenna, microstrip antenna, microstrip short backfire antenna, short backfire antenna

\section{INTRODUCTION}

The short backfire antenna (SBFA) is known since of 1960s [1], [2]. It consists of two parallel plate reflectors with different dimensions (a big and small reflector), spaced a distance about $\lambda / 2$ apart, and a driven element, usually $\lambda / 2$ dipole placed about midway between the reflectors. Because of its compact construction and high performance the SBFA is very popular today, and is widely used in mobile satellite communications, tracking, telemetry, and wireless local-area network systems [3], [4].

The microstrip short backfire antenna (MSSBFA) combines a microstrip-type element (patch element) with a backfire cavity [5]. In this antenna construction the patch element can be considered as a feed of the backfire cavity. The areas between the big reflector and the patch, and between the patch and the small reflector are filled by different dielectrics named patch and small reflector substrate, respectively. In order to achieve a maximum gain the both reflectors of the MSSBFA are circular. For achievement of a high polarization purity in the case of linear polarization the shape of the patch is chosen rectangular. The gain of the MSSBFA is more than three times (about $4.8 \mathrm{dBi}$ ) the gain of a single microstrip antenna (MSA) and it can provide a gain of about $11 \mathrm{dBi}$. The described antenna has a coaxial feed which limits its applications as an element of complex antenna arrays. However its main disadvantage is the narrow bandwidth less than several per cent [6] due to the resonant structure and the impedance mismatch between the open antenna cavity and the free space. In addition the circular shape of the small reflector decreases the cross-polarization characteristics of the antenna.

Wideband MSSBFAs with three-layer radiating part including a patch substrate, an air gap, and a small re- flector substrate are proposed in [7] and [8]. These antenna constructions have a wide bandwidth (approximately $20 \%$ ) but their gain decreases to $7 \mathrm{dBi}$ (a value close to the gain of the conventional MSA providing about $6.5 \mathrm{dBi}$ ) at the resonant frequency. Except the coaxial feed and the impedance mismatch between the cavity and the free space they have low mechanical performance due to the presence of an air gap in their construction.

The goal of this study is a design and investigation of a simple, robust and compact MSSBFA with high gain and wide bandwidth.

\section{DESCRIPTION OF THE ANTENNA AND ITS MECHANISM OF RADIATION}

Figure 1 shows the geometry of the aperture coupled microstrip short backfire antenna (ACMSSBFA) and its dimensions are listed in Tab. 1. The antenna consists of the following elements: small reflector $R_{1}$, ground plane $G P /$ big reflector $R_{2}$, patch $P$, rim $R$, slot (aperture) $S l$, microstrip feed line $M S F L$, screen $S$, connector $C$ and five substrates as follows: additional substrate $A S$ (Taconic TLX-7: $\varepsilon_{r t}=2.6, \tan \delta_{t}=0.0019$ ); small reflector substrate $S R S$ (Arlon AD 410: $\varepsilon_{r q}=4.1$, $\tan \delta_{q}=0.0030$, the small reflector substrate is realized by two layers with standard thickness of $3.175 \mathrm{~mm}$ ); patch substrate $P S$ (Arlon $\mathrm{AD}$ 600: $\varepsilon_{r P}=6.15, \tan \delta_{P}=$ 0.0030); feed substrate FS (Arlon AD 600: $\varepsilon_{r f}=6.15$, $\tan \delta_{f}=0.0030$ ) and screen substrate SS (Taconic TLX$\left.7: \varepsilon_{r S}=2.6, \tan \delta_{S}=0.0019\right)$.

The radiation mechanism of the antenna is the following: the electromagnetic energy provided by the feed microstrip line penetrates via the slot cut in the ground plane into the patch resonator formed by the patch and the ground plane. The electromagnetic energy from this

\footnotetext{
* Department of Radioengineering of the Technical University of Varna, Studentska Str. 1, 9010 Varna, Bulgaria, gkirov@abv.bg, lz2gec@abv.bg ${ }^{* *}$ Navigat Ltd, Varna
} 


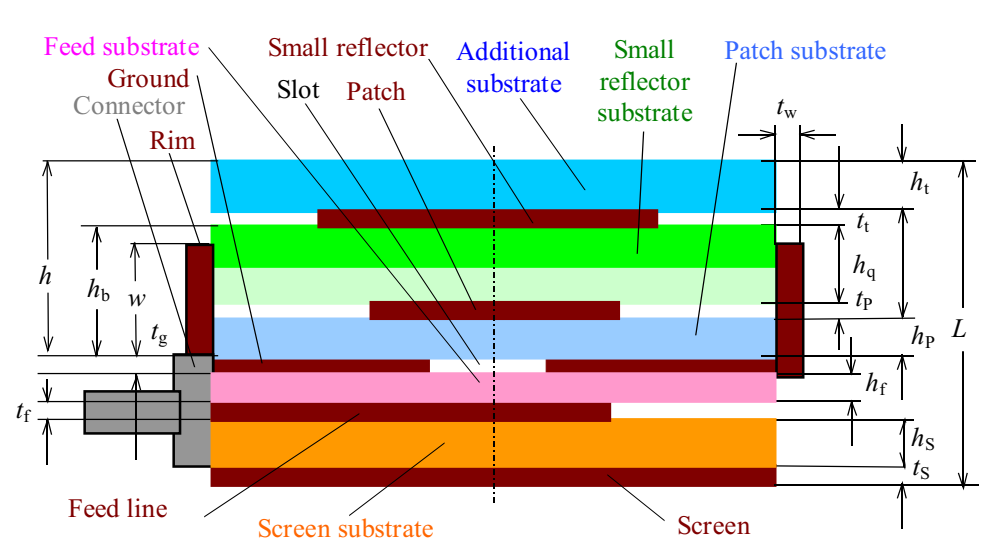

(a)

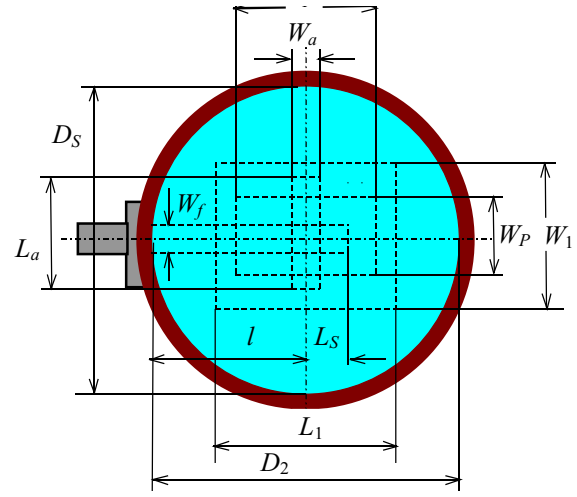

(b)

Fig. 1. Geometry of the antenna: (a) cross section, (b) front view

Table 1. Dimensions of the antenna

\begin{tabular}{ccl}
\hline \multicolumn{2}{c}{ Dimension } & Description \\
\hline$D_{2}$ & 24 & Big reflector inner diameter \\
$t_{g}$ & 0.0175 & Big refl. \& Ground plane thick. \\
$L$ & 13.145 & Antenna length \\
$L_{1}$ & 7.6 & Small reflector length \\
$W_{1}$ & 6.175 & Small reflector width \\
$t_{t}$ & 0.035 & Small reflector thickness \\
$w$ & 6.1 & Rim width \\
$t_{w}$ & 1.0 & Rim thickness \\
$L_{P}$ & 2.2 & Patch length \\
$W_{P}$ & 1.788 & Patch width \\
$t_{P}$ & 0.035 & Patch thickness \\
$L_{a}$ & 3.8 & Slot (aperture) length \\
$W_{a}$ & 0.38 & Slot (aperture) width \\
$l$ & 12 & Microstrip feed line length \\
$L_{S}$ & 0.7 & Stub length \\
$W_{f}$ & 0.98 & Microstrip feed line width \\
$t_{f}$ & 0.0175 & Microstrip feed line thickness \\
$D_{S}$ & 24 & Screen diameter \\
$t_{S}$ & 0.035 & Screen thickness \\
$h_{t}$ & 1.575 & Additional substrate thickness \\
$h_{q}$ & 6.35 & Small refl. substrate thickness \\
$h_{P}$ & 1.27 & Patch substrate thickness \\
$h_{f}$ & 0.635 & Feed substrate thickness \\
$h_{S}$ & 3.175 & Screen substrate thickness \\
\hline & &
\end{tabular}

resonator is radiated by the couple of nonresonant slots formed between the shorter sides (with dimension $W_{P}$ ) of the patch and the ground plane in the broadside direction of the antenna. One part of this energy is radiated directly into the space and the other part excites the second resonator (the backfire resonator) of the antenna formed by the small reflector and the ground plane. After multiple reflections from the both reflectors of the backfire resonator a large amount of its energy is also radiated via the aperture formed between the small reflector and the rim in the broadside of the antenna. The presence of the rim whose width is about $\lambda / 4$ decreases the back and the side radiations of the antenna.

\section{DESIGN OF THE ANTENNA}

The first step of the antenna design is carried out according to the recommendations of [9] reducing all dimensions approximately $n=\sqrt{\varepsilon_{r}}$ times, where $\varepsilon_{r}$ is the relative dielectric constant of the corresponding substrate and $n$ is its index of refraction.

In order to obtain a maximum antenna gain and wide frequency bandwidth the following approaches and techniques are applied in the further design of the ACMSSBFA:

1) Type of the feed. From the four most popular techniques to feed the MSAs (microstrip line, coaxial probe, aperture coupling and proximity coupling) an aperture coupling feed is chosen for the antenna. This feed technique allows independent optimization of the feed and radiating parts of the antenna due to the metal ground plane placed between them;

2) Value of the radiating part substrate dielectric constant. It is known that the optimum value of the big reflector diameter of the MSSBFA is

$$
D_{2} \approx 2 \lambda_{\text {obeff }}=2 \lambda_{0} b / n_{\text {beff }}
$$

where $\lambda_{0 b e f f}$ and $\lambda_{0 b}$ are the wavelengths in the radiating part of the antenna and in the free space, respectively, corresponding to the backfire resonant frequency $f_{0 b}$. When the MSSBFA is used as an element of an antenna array, the diameter $D_{2}$ must be equal to its interelement spacing $d$ and it should be chosen in compliance with the following inequality

$$
0.5 \lambda_{0}<D_{2}<\lambda_{0}
$$

where $\lambda_{0}$ is the midband wavelength of the antenna corresponding to the central (operating) frequency $f_{0}$. The lower limit of (2) restricts the mutual coupling between the antenna array elements while the upper one limits the appearance of grating lobes in the antenna radiation pattern. Usually $f_{0 b}=f_{0}$ or $f_{0 b} \approx f_{0}$. Then the common solution of (1) and (2) gives

$$
2<n_{\text {eff }}<4
$$


or

$$
4<\varepsilon_{\text {reff }}<16
$$

Expression (4) signifies that the value of $\varepsilon_{r}$ of the MSSBFA with homogeneous substrate of its radiating part should be more than about 5 . On the other hand the MSA produce maximum gain and radiation efficiency when the same value is very low, for example $2-3$, even close to 1 (MSAs with a foam as a radiating part substrate). These contrary requirements do not allow a creation of high efficiency MSSBFA with homogeneous radiating part substrate;

3) Type of the radiating part substrate. The above analysis shows that the high efficiency MSSBFA should have an inhomogeneous substrate. Three different substrates are chosen in this antenna construction. For good impedance matching with the free space their indices of refraction must satisfy the condition

$$
n_{P}>n_{q}>n_{t}>1
$$

where $n_{P}, n_{q}$ and $n_{t}$ are the indices of refraction of the patch, small reflector and additional substrate, respectively, while 1 is the index of refraction of the free space. The best impedance matching is obtained when the neighbouring substrate ratios of indices of refraction are approximately equal

$$
n_{P} / n_{q}=n_{q} / n_{t}=n_{t} / 1
$$

4) Number of the resonances. In the conventional MSSBFA the patch and the backfire cavities are tuned at the same frequency. This coincidence of the both resonant frequencies assures a high antenna gain, but narrow impedance bandwidth. In this design the antenna bandwidth is widened by insertion of two close resonances in the return loss characteristic of the antenna [10]. The first one is the patch resonance, and the second one is the backfire resonance. In this manner the impedance bandwidth of the antenna is widened more than two times. Attempts, unfortunately not successful, were done to insert a third resonance - the slot resonance. In the last case the antenna tuning becomes more complex and unstable;

5) Shape of the patch and small reflector. For high antenna performance the shape of the patch and small reflector is chosen the same. For achievement of a high polarization purity in the case of linear polarization a rectangular shape is chosen and the values of the patch ratio $K_{P}=W_{P} / L_{P}$ and small reflector ratio $K_{1}=$ $W_{1} / L_{1}$ are fixed between 0.750 and 0.875 [11];

6) Inserting of a screen. A metal screen in the feed part of the antenna is involved. It reflects the back radiated energy from the slot in the direction of the basic radiated energy flux of the antenna improving its gain [12]. The value of the screen substrate index of refraction $n_{S}$ and thickness $h_{S}$ must satisfy the following condition

$$
n_{S} h_{S} \approx 0.25 \lambda_{0} n_{f} h_{f} t_{f}
$$

where $t_{f}$ is the thickness of the microstrip feed line metallization, and $n_{f}$ and $h_{f}$ are the feed substrate index of refraction and thickness, respectively;

7) Optimization of the antenna dimensions. A suitable choice of standard substrate dielectric constants and thicknesses is done. All other antenna dimensions are optimized by a computer simulation.

\section{EFFECT OF THE DIMENSIONS OF THE ANTENNA ON ITS ELECTRICAL CHARACTERISTICS}

The simulation of the antenna model is carried out by the software package HFSS v 11.1. The final results are verified once again using the software CST Microwave Studio 2008. As criteria in the optimization the impedance bandwidth and the gain are chosen.

Eight antenna dimensions are chosen in the optimization. In order to simplify the optimization procedure their number is reduced to five using the following interrelations: the patch width $W_{P}=K_{P} L_{P}, K_{P}=0.8125$; the slot width $W_{a}=K_{a} L_{a}$, where $K_{a}=W_{a} / L_{a}$ is a slot (an aperture) ratio, $K_{a}=0.1$ [11], and the small reflector width $W_{1}=K_{1} L_{1}, K_{1}=0.8125$. Finally, the following five antenna dimensions remain as independent parameters in the optimization: 1) Patch length $L_{P}$ : it influences on the patch resonance; 2) Slot length $L_{a}$ : this dimension acts on the coupling level between the feed energy and the patch cavity; 3) Small reflector length $L_{1}$ : it determines the radiating aperture dimension of the antenna; 4) Stub length $L_{S}$ : this dimension influences on the impedance matching and the frequency bandwidth of the antenna; 5) Rim width $w$ : it acts on the level of the back and side lobe radiations of the antenna.

Five electrical characteristics are investigated in the frequency range $11 \mathrm{GHz}$ to $13.5 \mathrm{GHz}$ in the simulation: return loss, back radiation, directivity, gain and radiation patterns.

Figures 2 to 6 show the return loss (the module of the reflection coefficient $S_{11}$ ) and the gain of the simulated antenna model versus frequency.

It is seen from the figures a strong influence of the all five dimensions on the return loss characteristic, while the same dimensions act insignificantly on the antenna gain. The weak influence of the patch length, slot length and stub length on the antenna gain is expressed mainly in the low frequency part of the antenna bandwidth.

Figure 7 shows the E- and H-plane co-polar (solid line) and cross-polar (dashed line) radiation patterns of the antenna at $f=12.4 \mathrm{GHz}$. The antenna has a very good cross- polarization characteristic in H-plane. The H-plane radiation pattern possesses lower levels of the side lobes and the cross-polarization than the radiation pattern in E-plane. The asymmetry in the E-plane radiation pattern is due to the presence of the antenna connector (SMA type connector).

Figures 8 and 9 show the VSWR, the back radiation, the directivity and the gain versus frequency calculated 


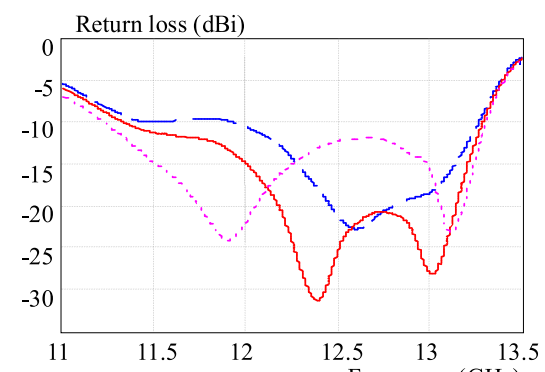

(a)

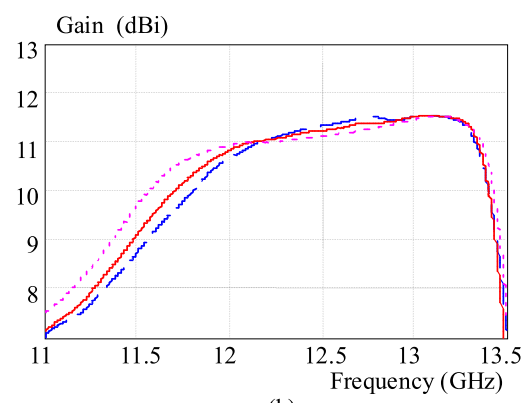

(b)

Fig. 2. Effect of the patch length $L_{P}$ $\left(L_{P}=1.9 \mathrm{~mm}-\right.$ dashed line, $L_{P}=$ $2.2 \mathrm{~mm}-$ solid line and $L_{P}=2.5 \mathrm{~mm}-$ dotted line) on the electrical characteristics of the antenna: (a) - return loss, (b) gain

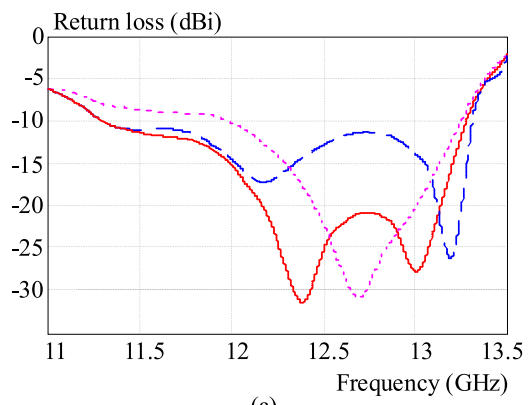

(a)

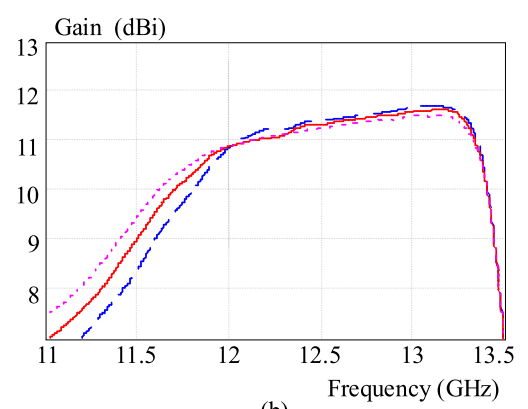

(b)

Fig. 5. Effect of the stub length $L_{S}$ $\left(L_{S}=0.5 \mathrm{~mm}-\right.$ dashed line, $L_{S}=$ $0.7 \mathrm{~mm}$ - solid line and $L_{S}=0.9 \mathrm{~mm}-$ dotted line) on the electrical characteristics of the antenna: (a) - return loss, (b) gain
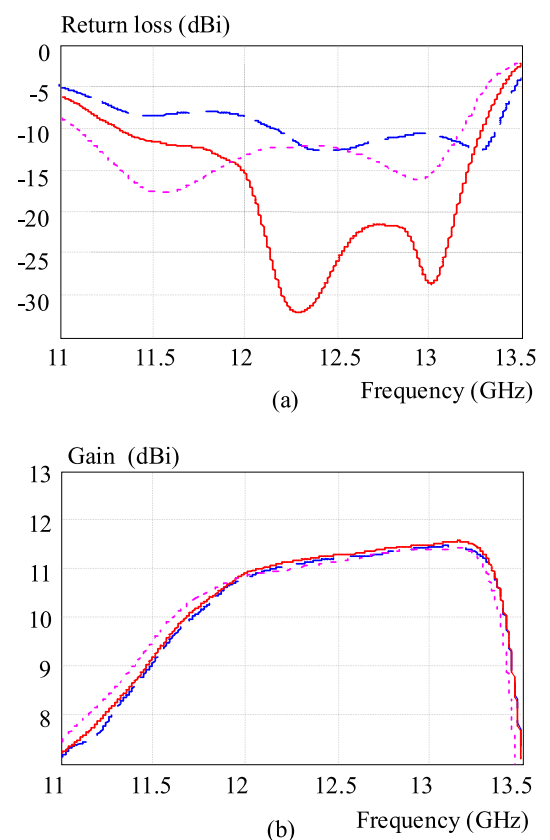

Fig. 3. Effect of the slot length $L_{a}\left(L_{a}=\right.$ $3.6 \mathrm{~mm}$ - dashed line, $L_{a}=3.8 \mathrm{~mm}-$ solid line and $L_{a}=4 \mathrm{~mm}$ - dotted line) on the electrical characteristics of the antenna: (a) - return loss, (b) - gain

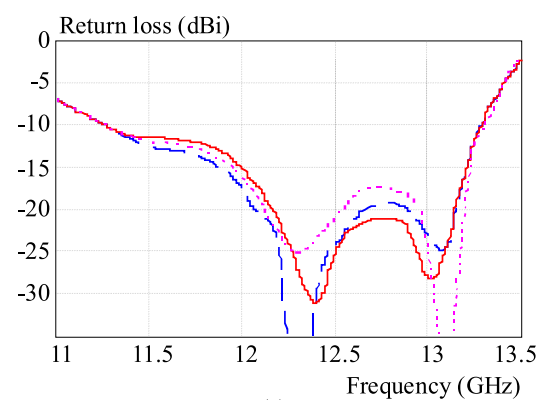

(a)

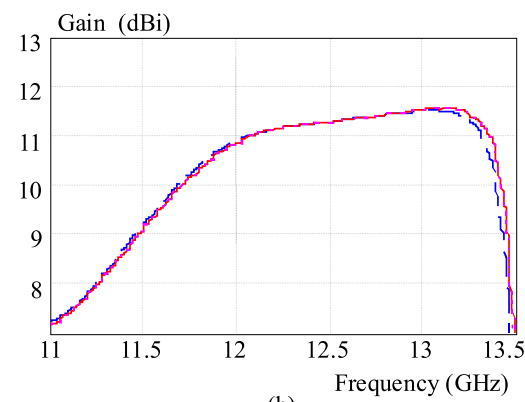

(b)

Fig. 6. Effect of the rim width $w(w=$ $5.9 \mathrm{~mm}$ - dashed line, $w=6.1 \mathrm{~mm}-$ solid line and $w=6.3 \mathrm{~mm}$ - dotted line) on the electrical characteristics of the antenna: (a) - return loss, (b) - gain
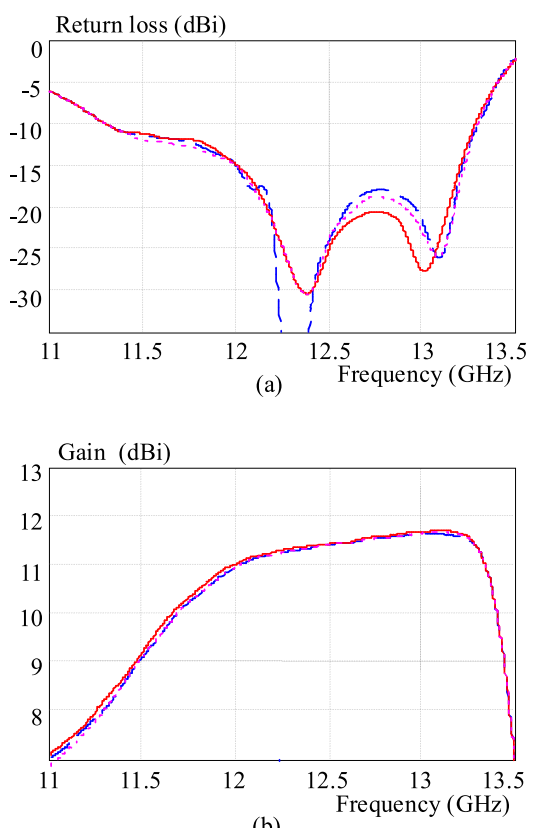

(b)

Fig. 4. Effect of the small reflector length $L_{1} \quad\left(L_{1}=7.2 \mathrm{~mm}-\right.$ dashed line, $L_{1}=$ $7.6 \mathrm{~mm}$ - solid line $L_{1}=7.8 \mathrm{~mm}$ - dotted line) on the electrical characteristics of the antenna: (a) - return loss, (b) - gain

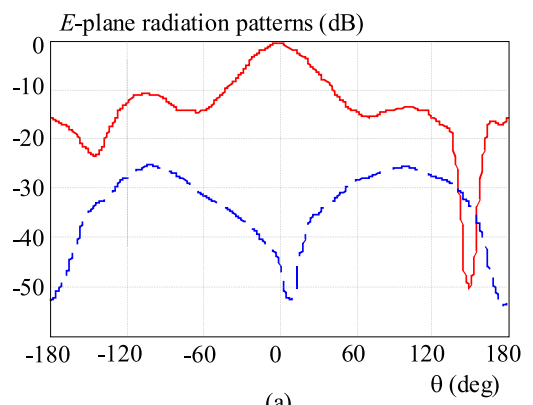

(a)

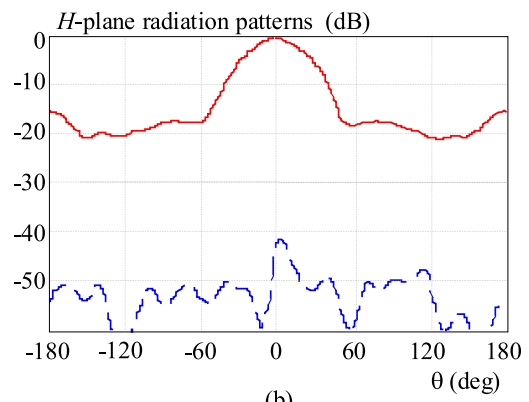

(b)

Fig. 7. Radiation patterns of the antenna (co-polar - solid line and cross-polar dashed line), $f=12.4 \mathrm{GHz}$ : (a) $-E$ plane, (b) $-H$-plane by means of two different softwares: HFSS v 11.1 (solid line) and CST Microwave Studio 2008 (dashed line). Except the back radiation, all other characteristics do not differ by more than $0.5 \mathrm{~dB}$ which may be considered as a good simulation result.

\section{SIMULATION RESULTS}

Several iterations are done to find the optimum values of the eight variable antenna dimensions: $L_{P}=$ $2.2 \mathrm{~mm}, W_{P}=1.788 \mathrm{~mm}, L_{a}=3.8 \mathrm{~mm}, W_{a}=0.38 \mathrm{~mm}$, 


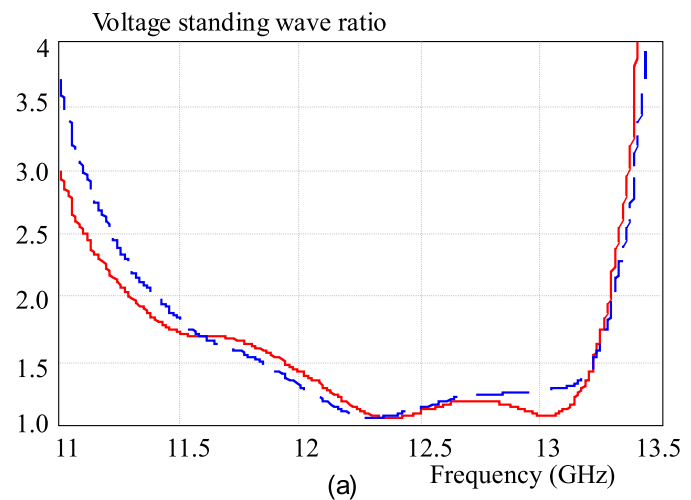

Back radiation level $(\mathrm{dB})$

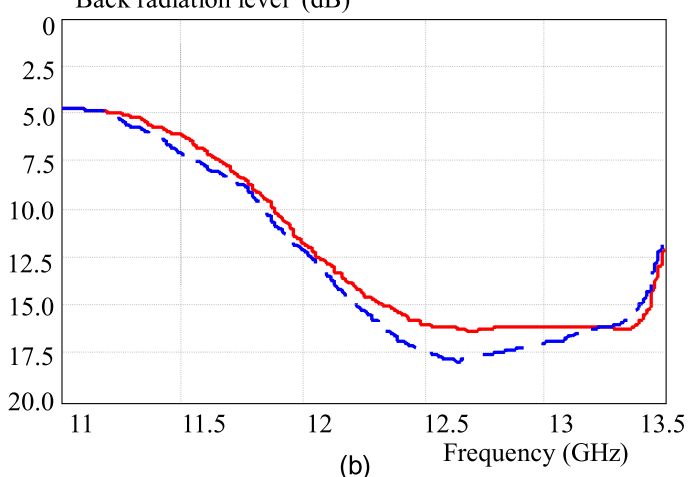

(b)

Fig. 8. VSWR and back radiation level of the antenna: HFSS v 11.1 — solid line, CST MWS 2008 - dashed line: (a)-VSWR, (b)- back radiation level

$L_{1}=7.6 \mathrm{~mm}, W_{1}=6.175 \mathrm{~mm}, L_{S}=0.7 \mathrm{~mm}$ and $w=6.1 \mathrm{~mm}$. All dimensions of the antenna after the optimization are given in Tab. 1.

The basic electrical parameters of the antenna obtained by simulation are summarized in Tab. 2 .

Three bandwidths of the antenna are defined [13]: impedance bandwidth (specified at a level of $-10 \mathrm{~dB}$ of the characteristic $S_{11}(f)$, directivity and gain bandwidths (specified at a level $3 \mathrm{~dB}$ less than their maximum values). The antenna bandwidth shown in Tab. 2 is defined as a frequency bandwidth within which the three above requirements are satisfied.

\section{DISCUSSION}

In this study an aperture coupled microstrip short backfire antenna was designed and investigated. The following features of the studied antenna construction need additional attention and discussion.

1) Advantages of the antenna. The main antenna advantage is the obtained wide bandwidth (of about 15\%). The maximum gain of the antenna is $11.5 \mathrm{dBi}$ and in a $77 \%$ of the bandwidth (from $11.88 \mathrm{GHz}$ to $13.28 \mathrm{GHz}$ ) the value of the antenna gain is more than $10.5 \mathrm{dBi}$. This is a good result compared to the conventional MSA $(G \approx 6.5 \mathrm{dBi})$ and the SBFA with an air cavity $(G \approx$ $13-15 \mathrm{dBi}$ ). The antenna construction is compact, robust and with a low volume. The volume and the aperture area of the antenna, for example, are 3.7 times less than the
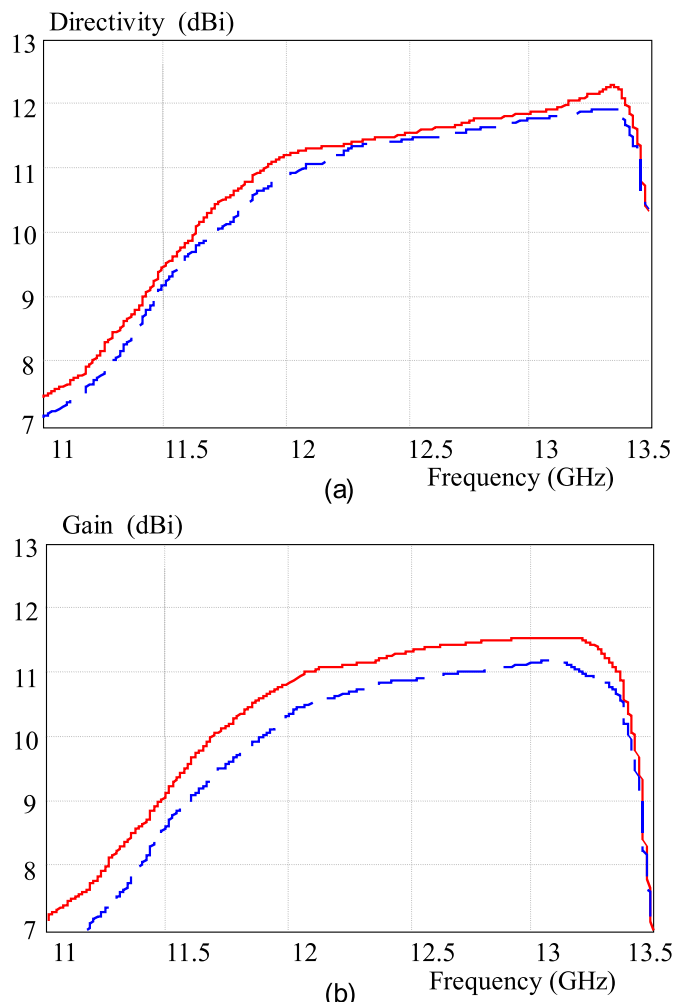

Fig. 9. Directivity and gain of the antenna: HFSS v 11.1 - solid line, CST MWS 2008 - dashed line: (a)- directivity, (b)- gain

corresponding dimensions of the SBFA with an air cavity while the lengths of the both antennas are approximately the same (about $0.54 \lambda_{0}$ ).

2) Disadvantages of the antenna. The main disadvantage of the antenna is its high level of the back radiation (BRL) in the low frequency part of the bandwidth $(-6 \mathrm{~dB}$ to $-10 \mathrm{~dB}$ over the frequency range from $11.47 \mathrm{GHz}$ to $11.88 \mathrm{GHz}$ or $23 \%$ of the antenna bandwidth). The decrease of the antenna $B R L$ will be the main task in the further investigations. In this manner some increase of the antenna gain could be expected in the same frequency range.

3) Inserting of two resonances. This is the main factor leading to widening of the antenna bandwidth. The first resonance at the lower frequency $\left(f_{0 P}=12.360 \mathrm{GHz}\right)$ is associated with the patch and the second one at the higher frequency ( $f_{0 b}=13.040 \mathrm{GHz}$ ) is connected to the backfire cavity. It is very important to be noted that the both resonances are not proper resonances but mutual resonances of the patch and the backfire cavity. This fact may be easy verified by simple calculations using the well known expressions for these resonant frequencies given in [6] and [3], respectively.

4) Type of the radiating substrate. The investigations carried out in this study show that a high efficiency MSSBFA may be created using an unhomogeneous radiating part substrate. In this case the dielectric constants (or the indices of refraction) of the used substrates should be chosen according to the requirements of expressions (5) and (6). 


\section{CONCLUSION}

A wideband, compact and robust aperture coupled microstrip short backfire antenna is designed and investigated in this study. It operates in the Ku-band $(12.375 \mathrm{GHz})$ and has a $15 \%$ frequency bandwidth. The maximum gain of the antenna is $11.5 \mathrm{dBi}$ and in a $77 \%$ of the bandwidth the value of its gain is more than $10.5 \mathrm{dBi}$. The volume and the aperture area of the antenna are approximately 3.7 times less than the corresponding dimensions of the conventional short backfire antenna with an air cavity. The antenna may find various applications as a single radiator and in antenna arrays in the contemporary communication systems when wide bandwidth, compactness and light weight are important.

\section{Acknowledgment}

The authors wish to acknowledge the Bulgarian Ministry of Education, Youth and Science and the Technical University of Varna for financial support under the Research Project No PD-2/2010"Study of Microstrip Short Backfire Antennas".

Table 2. Electrical parameters of the antenna

\begin{tabular}{lc}
\hline Electrical parameter & Value \\
\hline Minimum frequency $f_{\min }, \mathrm{GHz}$ & 11.470 \\
Maximum frequency $f_{\max }, \mathrm{GHz}$ & 13.280 \\
Central frequency $f_{0}, \mathrm{GHz}$ & 12.375 \\
Central wavelength $\lambda_{0}, \mathrm{~mm}$ & 24.242 \\
Frequency bandwidth $B W, \mathrm{GHz}$ & 1.810 \\
Fractional bandwidth $b w, \%$ & 14.63 \\
Maximum directivity $D_{\max }, \mathrm{dBi}$ & 12.1 \\
Directivity in the bandwidth $D, \mathrm{dBi}$ & $>9.1$ \\
Maximum gain $G_{\max }, \mathrm{dBi}$ & 11.5 \\
Gain within the bandwidth $G, \mathrm{dBi}$ & $>9$ \\
Max. radiation efficiency $\eta_{\mathrm{effmax}}, \%$ & 95.5 \\
Rad. efficiency in the bandwidth $\eta_{\mathrm{eff}}, \%$ & $>82.1$ \\
Max. back radiation level $B R L_{\mathrm{max}}, \mathrm{dB}$ & -6 \\
\hline \multicolumn{1}{c}{ Radiation patterns at $f=12.400 \mathrm{GHz}$} \\
\hline E-plane beamwidth $2 \theta_{3 \mathrm{dBE}}, \mathrm{deg}$ & 40 \\
Max. E-plane sidelobe $S L L_{\mathrm{Emax}}, \mathrm{dB}$ & -10 \\
Max. E-plane cross-pol $X P L_{\mathrm{Emax}}, \mathrm{dB}$ & -25 \\
H-plane beamwidth $2 \theta_{3 \mathrm{dBH}}, \mathrm{deg}$ & 46 \\
Max. H-plane sidelobe $S L L_{\mathrm{H} \max }, \mathrm{dB}$ & -17 \\
Max. H-plane cross-pol $X P L_{\mathrm{Hmax}}, \mathrm{dB}$ & -44 \\
Back radiation level $B R L, \mathrm{~dB}$ & -15 \\
\hline
\end{tabular}

\section{REFERENCES}

[1] TRENTINI, G. V.: Bündelung elektrischer Wellen durch Leitscheiben, Z. angew. Physik 6 No. 10 (1954), 462-470.

[2] EHREnspeCK, H. W.: The Short Backfire Antenna, Proc. IEEE 53 No. 8 (1965), 1138-1140.

[3] KUMAR, A.-HRISTOV, H. D.: Microwave Cavity Antennas, Artech House, Norwood, MA, 1989.
[4] Fujimoto, K.-JAmes, J. R.: Mobile Antenna Systems Handbook, Second Edition, Artech House, Norwood, MA, 2001.

[5] KALOI, C. M.-HATFIELD, D.-SIMON, P.: Microstrip Backfire Antenna, Proceedings IEEE AP-S Symposium, 1981, pp. 343-346.

[6] BALANIS, C. A. : Modern Antenna Handbook, A John Wiley \& Sons, Inc., 2008.

[7] LEE, R. Q.-LEE, K. F. : Experimental Study of the Two-Layer Electromagnetically Coupled Rectangular Patch Antenna, IEEE Trans. on AP 38 No. 8 (1990), 1298-1302.

[8] NISHIYAMA, E.-AIKAWA, M.: FDTD Analysis of Stacked Microstrip Antenna with High Gain, Progress In Electromagnetics Research, PIER 33 (2001), 29-43.

[9] KIROV, G. S. : Design of Short Backfire Antennas, IEEE Antennas \& Propagation Magazine 51 No. 6 (2009), 110-120.

[10] HRISTOV, H. D.-KIROV, G. S.: Short Backfire Antenna,, Certificate of Invention of R. P. Bulgaria 44853 Dec 1987.

[11] SAINATI, R. A.: CAD of Microstrip Antennas for Wireless Applications, Artech House, Norwood, MA, 1996.

[12] KIROV, G. S.-MIHAYLOVA, D. P.: Circularly Polarized Aperture Coupled Microstrip Antenna with Resonant Slots and a Screen, Radioengineering 19 No. 1 (2010), 111-116.

[13] POZAR, D. M.-SCHAUBERT, D. H. : Microstrip Antennas, IEEE Press, 1995.

Received 27 September 2010

Georgi Kirov (Associate Professor in communication engineering at the Department of Radioengineering, Technical University of Varna) was born in Malko Tarnovo, Bulgaria, in 1945. He received his MSc and $\mathrm{PhD}$ degrees in radio engineering from the Technical University of Varna, Bulgaria in 1968 and from the Technical University of Sofia in 1980, respectively. In 1969, he joined the Department of Radioengineering and, in 1992 became the head of the department. He specialized in microwave antennas at Moscow Technical University of Communications and Informatics in 1973 and 1978 and at the University of Luxembourg in 2006. He was also a visiting professor at the Otto von Guericke University of Magdeburg, Germany in 2002-2003. Dr Kirovs name is listed in some American and Great Britain encyclopedias such as Marquis Whos Who in the World, Marquis Whos Who in Science and Engineering and 2000 Outstanding Intellectuals of the $21^{\text {st }}$ Century (IBC Cambridge, England). He is a member of IEEE. His research interests include high frequency electromagnetism, antennas and wave propagation.

Georgi Chervenkov was born in Gorna Oriahovitsa, Bulgaria, in 1978. He received his MSc degree in telecommunication engineering from the National Military University Veliko Tarnovo, Bulgaria in 2001. He has over seven years experience in the field of microwave radio relay and optical link maintenance. He is currently a $\mathrm{PhD}$ student at the Department of Radioengineering, Technical University of Varna. Mr. Chervenkov is a member of IEEE and IET. His research interests include design, analysis and optimization of microstrip high-gain antennas and arrays.

Chavdar Kalchev was born in Tutrakan, Bulgaria, in 1977. He received his MSc degree in electronics from the Technical University of Varna, Bulgaria in 2001. In 2002, he joined the company Navigat LTD, Varna. He is currently a PhD student at the Department of Electronic Engineering and Microelectronics, Technical University of Varna. His research interests include studies in the field of digital signal processing and radar systems. 